\title{
Hemangioma of the Cavernous Sinus: A Case Series
}

\author{
Dylan A. Noblett ${ }^{1}$ Jennifer Chang ${ }^{1}$ Atrin Toussi ${ }^{2}$ Arthur Dublin ${ }^{1} \quad$ Kiarash Shahlaie $^{2}$ \\ ${ }^{1}$ Departments of Radiology, University of California, Davis Medical \\ Center, Sacramento, California, United States \\ 2 Departments of Neurological Surgery, University of California, \\ Davis Medical Center, Sacramento, California, United States
Address for correspondence Kiarash Shahlaie, MD, PhD, Department of Neurological Surgery, University of California, Davis Medical Center, 4860 Y Street, Suite 3740, Sacramento, CA 95817, United States (e-mail: krshahlaie@ucdavis.edu).

J Neurol Surg Rep 2018;79:e26-e30.

\begin{abstract}
Introduction Cavernous sinus hemangiomas (CSHs) are rare, vascular, extra-axial tumors that are diagnosed with a combination of imaging and biopsy. We describe the clinical presentations, imaging findings, and management of two male patients with CSHs.

Case Report Case 1 describes a 57-year-old man who presented with vision changes and cranial nerve palsies. Initial imaging and surgical biopsy were nondiagnostic. Follow-up Tc-99m tagged red blood cell (RBC) imaging supported CSH diagnosis. He was treated with surgical resection and radiotherapy.

Case 2 describes a 57-year-old man who presented with chronic headache. Imaging findings were suggestive of $\mathrm{CSH}$. He underwent endoscopic endonasal surgical resection and a final diagnosis of CSH was made via biopsy.

\section{Keywords}

- hemangioma

- cavernous sinus

- radiosurgery

- nuclear medicine

- neuroradiology

- neuroimaging

Discussion CSHs often present with headache, vision changes, and cranial nerve palsies. Characteristic findings of a T2 hyperintense lesion with homogeneous contrast enhancement has been described in the literature. There is also a role for tagged RBC imaging studies in the setting of nondiagnostic imaging and biopsy. Surgical resection can be difficult due to tumor vascularity and encasement of internal carotid arteries. Stereotactic radiosurgery and adjuvant radiotherapy can play a role in the treatment of patients who have inoperable lesions or subtotal resections.
\end{abstract}

\section{Introduction}

Cavernous sinus hemangiomas (CSHs) are rare, benign extraaxial tumors, primarily affecting middle-aged women. ${ }^{1}$ Symptoms vary, but are typically due to mass effect and include headache, vision changes, and cranial nerve palsies. In this case series, we describe the clinical presentation, imaging findings, and management of two male patients with CSHs encasing the cavernous segment of the internal carotid artery (ICA), making surgical resection challenging. Additionally, we report the use of Tc-99m tagged red blood cell (RBC) imaging to assist with diagnosis of cavernous hemangioma in the setting of nondiagnostic surgical biopsy.

\section{Case Reports}

\section{Case 1}

\section{Clinical Presentation}

CJ is a 57-year-old man who first presented to ophthalmology after noticing a progressive worsening and blurriness of vision in his left eye over the last 7 months. Neurological examination revealed mild anisocoria, left-sided ptosis, left-sided lid lag, diplopia with upward gaze, and hypesthesia in the distribution of the trigeminal nerve. A detailed eye exam demonstrated an inferior altitudinal visual field defect and a relative afferent pupillary defect in the left eye, concerning for ischemic optic received

October 5, 2017

accepted

March 2, 2018
DOI https://doi.org/

10.1055/s-0038-1641731. ISSN 2193-6358. (c) 2018 Georg Thieme Verlag KG
Stuttgart · New York

License terms

$\Theta(1) \Theta \Theta$ 


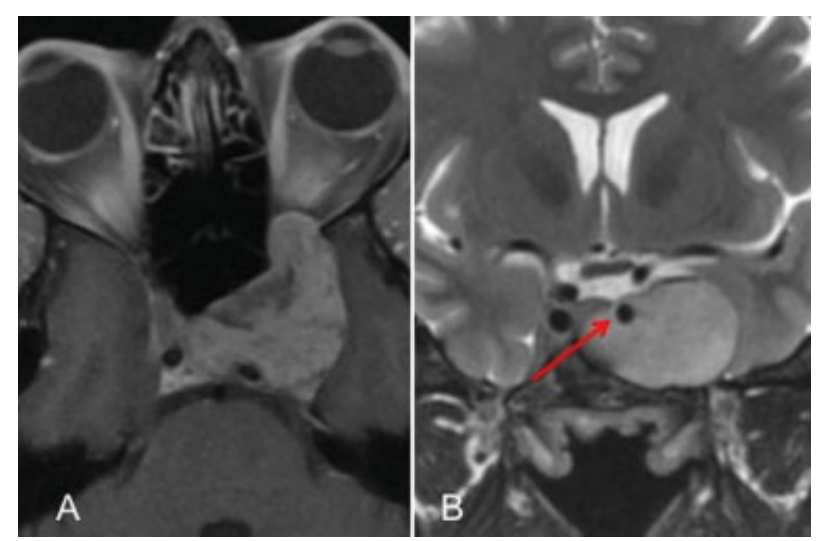

Fig. 1 CJ. Magnetic resonance imaging (MRI) of the brain with axial T1 postcontrast (A) and coronal T2 (B) weighted images of the sellar region demonstrates heterogeneous enhancement of a well-defined extra-axial mass centered in the left cavernous sinus with encasement of the left internal carotid artery flow void (red arrow), which remained patent.

neuropathy. The patient had no history of diabetes, but was a smoker of 10 years with hypertension managed with amlodipine and quinapril.

\section{Imaging Studies}

Magnetic resonance imaging (MRI) scan of the brain (-Fig. 1) demonstrated an extra-axial left parasellar mass measuring $35 \times 41 \times 24 \mathrm{~mm}$. The lesion was iso- to hyperintense on T2weighted imaging with heterogeneous enhancement after contrast administration. The mass extended medially to involve the left cavernous sinus and encased the left paraclinoid ICA; however, there was preservation of the ICA flow void, and vessel caliber was normal. The mass displaced the pituitary gland without invasion. Superiorly, there was some compression of the temporal lobe, without T2 hyperintensity to suggest underlying parenchymal edema or subpial extension. Anteriorly, the mass surrounded the orbital apex and extended through the inferior orbital fissure, compressing the prechiasmatic optic nerve.
Computed tomography (CT) angiogram (-Fig. $\mathbf{2 C}$ ) demonstrated small, scattered arterially enhancing components laterally and centrally within the mass. The mass encased the left cavernous ICA without occlusion. The inferior medial aspect of the mass abutted the inferior right cavernous ICA, causing mild narrowing.

\section{Management}

The patient underwent a left frontotemporal craniotomy approach for surgical debulking of his mass. After elevating the bone flap, a high-speed drill was used to resect the lateral sphenoid wing and remove the anterior clinoid process in an extradural fashion. The lesion was then identified at the orbital fissure, extending toward the orbital apex and cavernous sinus. The mass was entered along its lateral margin, and found to be extremely hemorrhagic with multiple vascular spaces. After a subtotal resection, hemostasis was achieved and the bone and scalp flap were then closed. The patient reported improved visual acuity and resolution of diplopia immediately after surgery. He had an uneventful postoperative course, and was discharged home on postoperative day 2 .

Pathology results from the surgical specimen revealed minute fragments of fibrovascular, focally cellular tissue. Immunohistochemistry was noncontributory. A definitive diagnosis was not obtained, although a vascular lesion was favored.

After the patient had recovered from his operation, a cerebral angiogram was obtained to further explore a vascular etiology. This study revealed abnormal enhancement arterial flow from the proximal branches of the left ophthalmic, recurrent meningeal, and left middle cerebral artery to supply the mass. There was no vascular contribution from external carotid artery and its branches. No arteriovenous shunting was noted. Findings were inconsistent with meningioma or hemagiopericytoma, and suggested the possibility of a CSH. The patient then underwent nuclear medicine scintigraphy, with administration of $20.3 \mathrm{mCi}$ Tc-99m RBC (- Fig. 3A). Also known as a tagged RBC scan, this study is generally used to detect

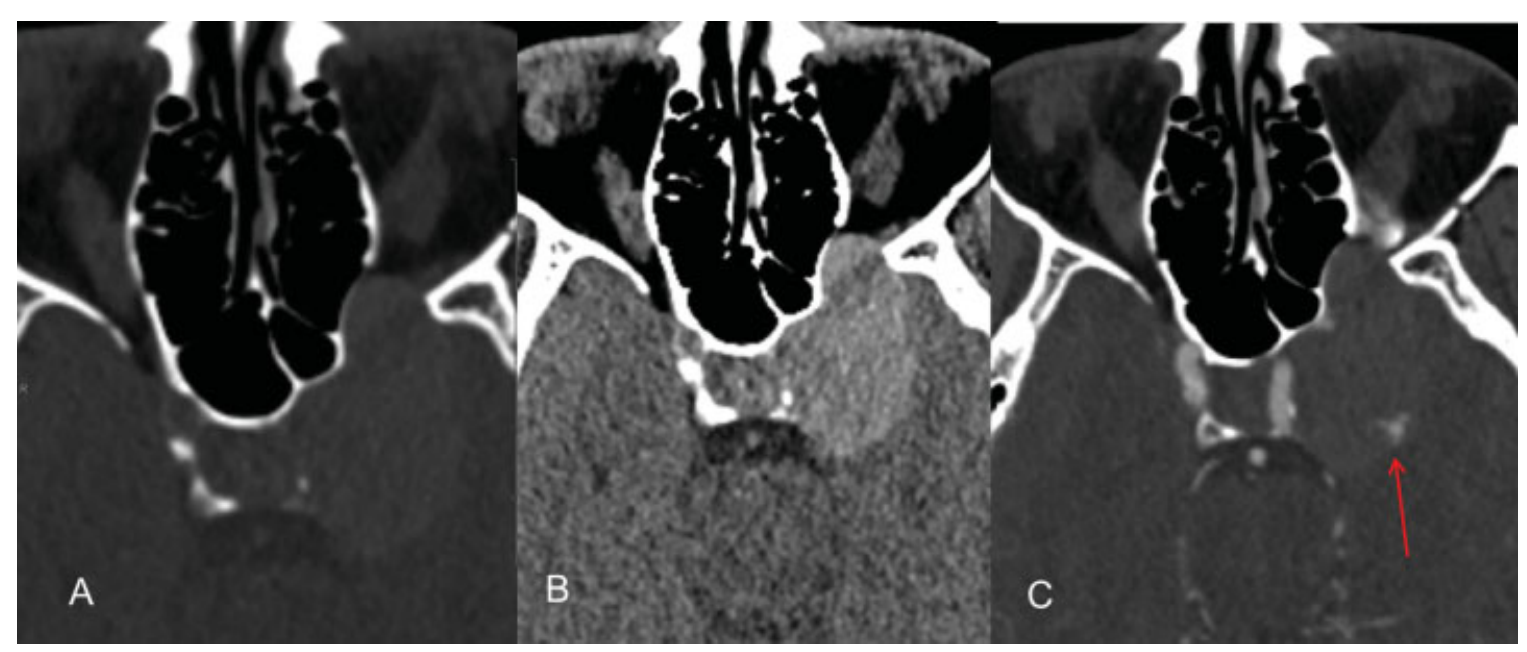

Fig. 2 CJ. Noncontrast computed tomography (CT) in bone (A) and brain (B) algorithm demonstrates a hyperdense mass centered in the left cavernous sinus extending into left orbital apex with smooth bony remodeling of the adjacent sphenoid sinus wall rather than destruction. $\mathrm{CT}$ angiogram (C) shows scattered arterially enhancing components within the periphery (red arrow). 


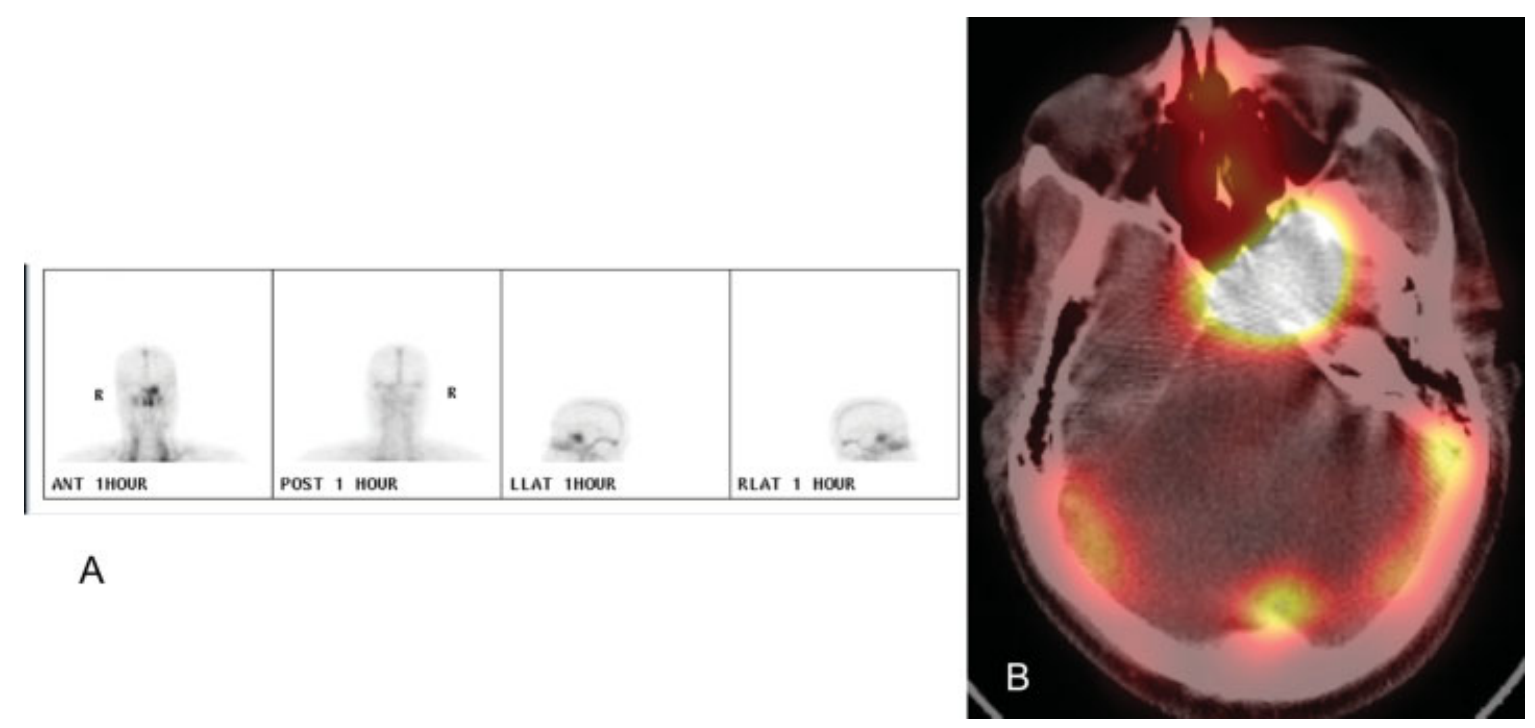

Fig. 3 CJ. Nuclear medicine tagged red blood cell scan. Delayed static images of the head after the administration of Tc-99m RBC (A) with anterior, posterior, and lateral views of the head demonstrate increased uptake within the left cavernous sinus mass. Single photon emission computed tomography (SPECT) (B) images of the head show avid increased uptake within the left cavernous sinus mass.

lower gastrointestinal (GI) bleeding; however, because erythrocytes are labeled with technetium-99m, lesions such as hemangiomas in the liver can also be confirmed, with increased activity on delayed blood pool images. Scintigraphy in addition to single photon emission computed tomography (SPECT) localization ( - Fig. 3B) revealed increased Tc-99m RBC activity within the known left cavernous sinus mass, highly suggestive of a hemangioma.

The patient went on to receive radiation therapy in the form of 46.8 Gy delivered over 26 fractions. Six months after radiation therapy, his vision has significantly improved and he has been able to return to work.

\section{Case 2}

\section{Clinical Presentation}

$\mathrm{MB}$ is a 57-year-old man who presented with a 6-year history of slowly progressive headache. Noncontrast head CT scan (-Fig. 4) demonstrated a 20-mm hyperdense mass within the left cavernous sinus that extended into the sella turcica and Meckel's cave, with mild mass effect on the medial temporal lobe. MRI scan (-Fig. 5) demonstrated heterogeneous peripheral enhancement and a central nonenhancing region. There was mass effect on the pituitary gland and stalk, and complete encasement of the cavernous segment of the ICA.

\section{Management}

The patient underwent an endoscopic endonasal transsphenoidal approach to the sella turcica and cavernous sinus. Intraoperative navigation and Doppler ultrasonography were used to identify the location of the ICA. The medial compartment of the cavernous sinus was opened, where a highly vascular, fibrotic tumor was encountered. The lesion was contained within the cavernous sinus, with compression, but no invasion, of the adjacent pituitary gland. After decompressing the cavernous sinus by resecting tissue medial to the ICA, the defect was repaired with a pedicled nasoseptal flap. The patient had an uneventful postoperative course, and was discharged home on postoperative day 1 .

Pathology results from the surgical specimen revealed fibrous tissue with numerous vascular channels, some containing organized thrombi. Differential diagnosis included angiomatous meningioma but the lack of epithelial membrane antigen (EMA) expression argued against this diagnosis. A final diagnosis was made of left CSH.

The patient initially refused adjuvant radiotherapy. He underwent surveillance imaging at 3, 6, and 12 months after surgery, during which time he remained asymptomatic. Approximately 18 months after surgery, repeat MRI demonstrated slight evidence of radiographic progression and the patient reported recent onset of mild facial dysesthesia. Physical examination at that time demonstrated mild ptosis with no diplopia or disconjugate gaze.

\section{Discussion}

\section{Cavernous Sinus Hemangioma}

CSHs are rare extra-axial intracranial tumors. They account for $\sim 3 \%$ of benign cavernous sinus masses and are more common in middle-aged women. ${ }^{1}$ The masses are typically slow-growing collections of thin-walled vascular channels that can exert mass effect on adjacent neurovascular structures. Pathologically, these masses can be divided into three major classifications: sponge-like, mulberry-like, and mixed. ${ }^{2}$ Sponge-like CSH typically possess an intact pseudocapsule and demonstrate homogenous contrast enhancement on MRI. In contrast, mulberry-like and mixed CSH have an incomplete or absent pseudocapsule and demonstrate heterogeneous contrast enhancement patterns. ${ }^{2}$ Unlike intra-axial cavernous hemangiomas, extra-axial CSH do not typically cause brain hemorrhage. ${ }^{3}$ 

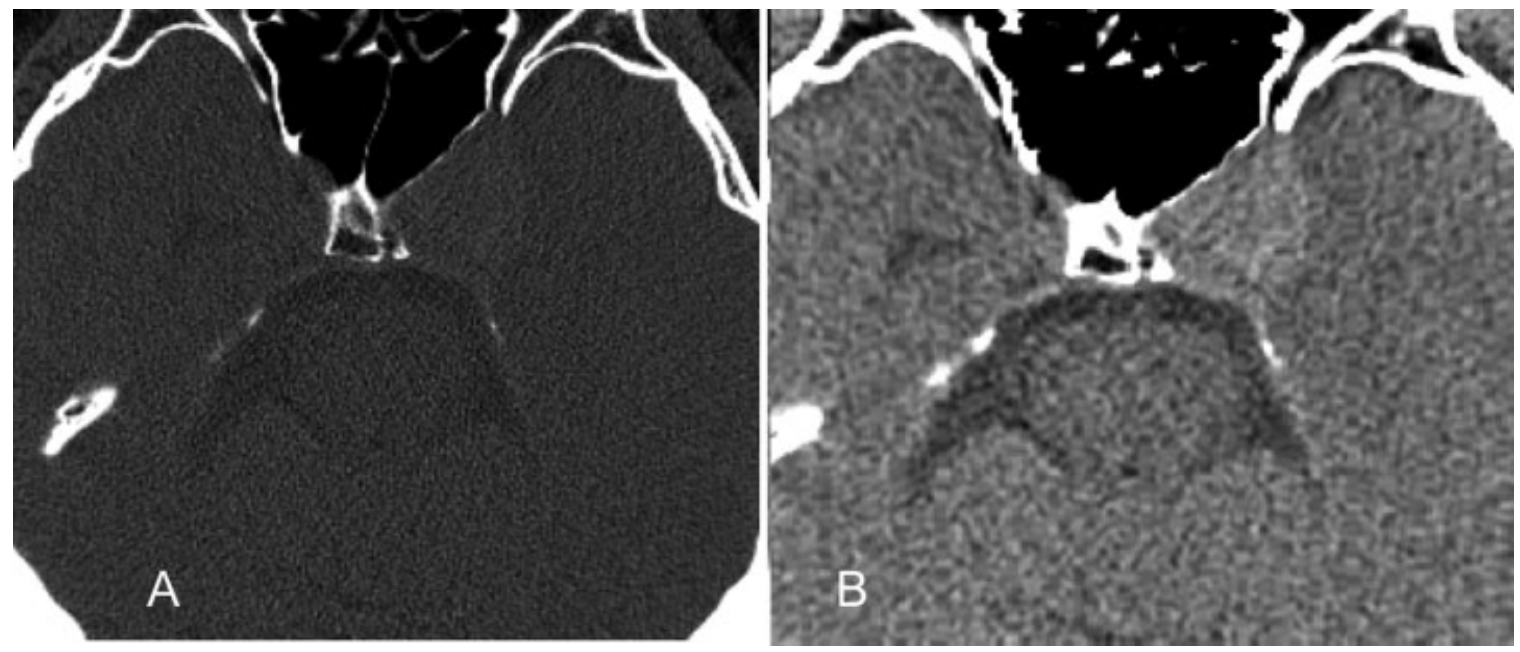

Fig. $4 \mathrm{MB}$. Noncontrast computed tomography $(\mathrm{CT})$ in bone $(\mathrm{A})$ and brain (B) algorithm demonstrates hyperdense mass centered in the left cavernous sinus with extension into the sella turcica and Meckel's cave with mild mass effect on the medial temporal lobe.

\section{Diagnostic Workup}

Hemangiomas only rarely occur in the cavernous sinus, where the differential diagnosis includes various neoplastic, infectious, inflammatory, and vascular etiologies., ${ }^{4,5}$ More common neoplastic lesions of the cavernous sinus are meningiomas and schwannomas, with chordomas, metastasis, lymphomas, and cavernous hemangiomas as rare possibilities. Common infectious and inflammatory etiologies include abscess, sarcoidosis, and Tolosa-Hunt syndrome. ${ }^{4}$ Vascular etiologies are typically cerebral aneurysms. Since the symptoms of cavernous sinus lesions are primarily due to mass effect impeding venous outflow and/or compressing cranial nerves, they are typically not specific and do not significantly focus the differential diagnosis.

MRI plays an important role in the workup of patients with CSH. These lesions typically demonstrate hypo- or isointensity on T1-weighted images and hyperintensity on $\mathrm{T} 2$-weighted images; these imaging findings alone lend a differential diagnosis of meningioma, schwannoma, pituitary adenoma, and CSH. MRI of CSH generally reveals T2 hyperintensity with some intratumor linear hypointenisty that is generally washed out by homogenous enhancement when intravenous contrast is used, likely representing thin walled vessels within the vascular malformation. ${ }^{3}$ CSHs generally appear more hyperintense than meningiomas and pituitary tumors, and the heterogeneous enhancement pattern of hemangiomas can differentiate them from the intense enhancement seen with schwannomas. Some studies have observed a hyperintense peripheral enhancement leading to a central hyperintensity pattern with timed contrast administration that is classically seen in hepatic cavernous hemangiomas. ${ }^{6}$

Since the first patient in our study did not have a diagnostic biopsy, a Tc-99m RBC nuclear medicine study was obtained to further characterize the lesion and ultimately make the diagnosis. We observed a dramatic increase in tracer accumulation in the left parasellar region, which is consistent with previous case reports utilizing this technique to diagnose hemangioma. ${ }^{7-9}$ It has been suggested that this tracer accumulation, which labels erythrocytes, is specific to $\mathrm{CSH}$ and is not noted in patients found to have other extraaxial masses in this region, including meningiomas, schwannomas, chordomas, or chondrosarcomas. ${ }^{7,9}$ This practice of utilizing tagged $\mathrm{RBC}$ nuclear medicine studies may be useful in future cases where the diagnosis of CSH is unclear and the physician requires a diagnosis for surgical planning or definitive treatment.
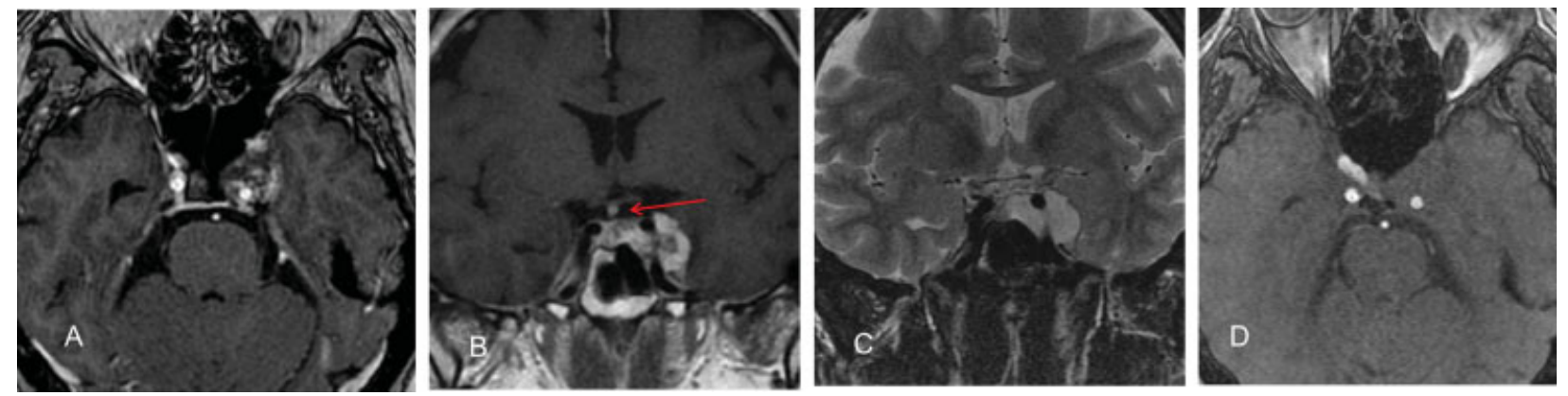

Fig. 5 MB. Axial T1 postcontrast (A) and coronal T1 postcontrast (B), images demonstrates heterogeneous peripheral enhancement with central nonenhancing portions of a left cavernous sinus mass with mass effect on the pituitary stalk (red arrow). Coronal T2 (C) images show a T2 hyperintense mass encasing the left internal carotid artery (ICA) flow void and axial magnetic resonance angiogram (MRA) (D) confirms normal flow related enhancement within the preserved ICA. 


\section{Management}

Surgical resection is the mainstay of treatment for hemangioma, reducing the likelihood of disease recurrence dramatically. When located in the cavernous sinus, however, the decision to perform complete surgical resection has to be carefully weighed against the risks of neurovascular injury, where up to $40 \%$ of tumors exhibit severe intraoperative bleeding. ${ }^{10}$ In one report, of 20 cavernous sinus cases, 7 were operated on using an intradural approach and within those 7 , one died of intracranial hemorrhage postoperatively. ${ }^{11}$ The most common postoperative complication is a transient ophthalmoplegia that develops in up to 40 to $86 \%$ of patients. $^{10,12}$ Rarely, permanent abducens nerve palsies can occur, with the incidence being $14 \%$ as reported in Suri et al's recent study. ${ }^{12}$ Morbidity and mortality after cavernous sinus surgery has generally been reported to be $38 \%$ in older reports and as high as $12.5 \%$ in more recent reports. ${ }^{13}$ As a result, many patients with cavernous sinus lesions may undergo subtotal resection followed by adjuvant therapies.

Stereotactic radiosurgery is a treatment option for patients with inoperable lesions or those that undergo limited subtotal resection. Gamma Knife radiosurgery (GKS) has been shown to significantly reduce tumor size and volume, as well as to relieve some of the neurological symptoms associated with $\mathrm{CSH} .{ }^{13}$ It is hypothesized that radiotherapy reduces lesion size by inducing endothelial proliferation, hyalinization of vessel walls, and cytotoxic effects of radiation on microvasculature and tumor cells themselves. Tang et al's 2015 study reported a $79 \%$ mean reduction in tumor volume in 53 patients treated with GKS during a 24.5-month follow-up period. Likewise, no tumor showed enlargement after treatment. ${ }^{14}$

\section{Conclusion}

CSHs are rare extra-axial benign tumors that often present with headache, diplopia, proptosis, or extraocular nerve palsies. MRI often demonstrates a T2 hyperintense lesion with homogeneous contrast enhancement with administration of contrast. Although imaging characteristics are often highly suggestive, in select cases Tc-99m tagged RBC nuclear medicine studies can be helpful in securing the diagnosis. Management of these lesions is directed by clinical presentation and tumor size, as aggressive surgical resection can have significant morbidity and is not indicated in patients with no significant cranial neuropathy. Radiation therapy plays a critical role in the management of these tumors, with excellent long-term control rates.

\section{Conflict of Interest}

None.

Acknowledgments

The authors did not receive any financial support for the work in this article.

\section{References}

1 Linskey ME, Sekhar LN. Cavernous sinus hemangiomas: a series, a review, and an hypothesis. Neurosurgery 1992;30(01):101-108

2 Yao Z, Feng X, Chen X, Zee C. Magnetic resonance imaging characteristics with pathological correlation of cavernous malformation in cavernous sinus. J Comput Assist Tomogr 2006;30 (06):975-979

3 Shi J, Hang C, Pan Y, Liu C, Zhang Z. Cavernous hemangiomas in the cavernous sinus. Neurosurgery 1999;45(06):1308-1313, discussion 1313-1314

4 Bag AK, Shah R. AJR teaching file: cavernous sinus mass in a woman presenting with painful ophthalmoplegia. AJR Am J Roentgenol 2010;195(3, Suppl):WS1-WS4

5 Razek AA, Castillo M. Imaging lesions of the cavernous sinus. AJNR Am J Neuroradiol 2009;30(03):444-452

6 Anqi X, Zhang S, Jiahe X, Chao Y. Cavernous sinus cavernous hemangioma: imaging features and therapeutic effect of Gamma Knife radiosurgery. Clin Neurol Neurosurg 2014;127:59-64

7 Salanitri GC, Stuckey SL, Murphy M. Extracerebral cavernous hemangioma of the cavernous sinus: diagnosis with MR imaging and labeled red cell blood pool scintigraphy. AJNR Am J Neuroradiol 2004;25(02):280-284

8 Kawai K, Fukui M, Tanaka A, Kuramoto S, Kitamura K. Extracerebral cavernous hemangioma of the middle fossa. Surg Neurol 1978;9(01):19-25

9 Sayit E, Durak I, Capakaya G, Yilmaz M, Durak H. The role of Tc$99 \mathrm{~m}$ RBC scintigraphy in the differential diagnosis of orbital cavernous hemangioma. Ann Nucl Med 2001;15(02):149-151

10 Yin YH, Yu XG, Xu BN, Zhou DB, Bu B, Chen XL. Surgical management of large and giant cavernous sinus hemangiomas. J Clin Neurosci 2013;20(01):128-133

11 Zhou LF, Mao Y, Chen L. Diagnosis and surgical treatment of cavernous sinus hemangiomas: an experience of 20 cases. Surg Neurol 2003;60(01):31-36, discussion 36-37

12 Suri A, Ahmad FU, Mahapatra AK. Extradural transcavernous approach to cavernous sinus hemangiomas. Neurosurgery 2007;60(03):483-488, discussion 488-489

13 Bansal S, Suri A, Singh M, et al. Cavernous sinus hemangioma: a fourteen year single institution experience. J Clin Neurosci 2014; 21(06):968-974

14 Tang $\mathrm{X}, \mathrm{Wu} \mathrm{H}$, Wang B, et al. A new classification and clinical results of Gamma Knife radiosurgery for cavernous sinus hemangiomas: a report of 53 cases. Acta Neurochir (Wien) 2015;157 (06):961-969, discussion 969 